\title{
New classes of harmonic meromorphic multivalent starlike functions in Janowski domain
}

\author{
Timilehin Gideon Shaba ${ }^{\mathrm{a}}$, Bakhtiar Ahmad ${ }^{\mathrm{b}}$, Muhammad Ghaffar Khan ${ }^{\mathrm{c}}$, Zabidin Salleh ${ }^{\mathrm{d}, *}$, Wali Khan \\ Mashwanic, Shahid Khan ${ }^{\mathrm{c}}$ \\ ${ }^{a}$ Department of Mathematics, University of Ilorin, P. M. B. 1515, Ilorin, Nigeria. \\ ${ }^{b}$ Government Degree College Mardan, 23200 Mardan, Pakistan. \\ ${ }^{c}$ Institute of Numerical Sciencies, Kohat University of Science and Technology, Kohat, Pakistan. \\ ${ }^{d}$ Department of Mathematics, Faculty of Ocean Engineering Technology and Informatics, Universiti Malaysia Terengganu, 21030 Kuala \\ Nerus, Terengganu, Malaysia.
}

\begin{abstract}
In this article some applications of harmonic analysis are discussed in the field of Geometric Function Theory in the form of a new class of meromorphic multivalent functions. A differential operator for these function have been used to define classes of meromorphic multivalent functions in association with Janowski functions in symmetric points. Various geometric properties like sufficiency criteria, growth theorem, convex combination, weighted mean for these functions have been evaluated for this newly defined class.
\end{abstract}

Keywords: Multivalent function, harmonic meromorphic function, Janowski function, starlike functions, subordination. 2020 MSC: 30C45, 30C50.

(C)2022 All rights reserved.

\section{Introduction and definitions}

Harmonic analysis have been utilized in the study of evaluating the solutions of fluid flow problems, (see [4, 10]). For instance, in 2012, Aleman and Constantin [4] built a relation between harmonic mappings and perfect flows of fluids. Recently, they have developed ingenious technique to solve the incompressible two dimensional Euler equations in terms of univalent harmonic mappings. More specifically, the issue of finding all solutions in which Lagrangian variables, defining the particle paths of the flow, presents a labeling by harmonic mappings and is then used to solve an explicit nonlinear differential system in $\mathrm{C}^{\mathrm{n}}$ (for more details see [10]). With more applied sciences its significance is of prime importance. Some of visible areas of latest interests for technologists would be quantum mechanics, signal processing, tidal

\footnotetext{
*Corresponding author

Email addresses: shabatimilehin@gmail.com (Timilehin Gideon Shaba), pirbakhtiarbacha@gmail.com (Bakhtiar Ahmad), ghaffarkhan020@gmail.com (Muhammad Ghaffar Khan), zabidin@umt.edu .my (Zabidin Salleh), mashwanigr8@gmail.com (Wali Khan Mashwani), shahidkhan@kust.edu.pk (Shahid Khan)
}

doi: $10.22436 /$ jmcs.024.03.03

Received: 2020-12-14 Revised: 2020-12-26 Accepted: 2021-01-03 
analysis and neuroscience. Keeping the above in sight we investigate some of its properties in connection with meromorphic multivalent functions in association with Janowski functions with Symmetric points.

A continuous complex valued function $f=u+i v$ is a harmonic function in complex domain $\mathcal{D}$ if both $u$ and $v$ are real harmonic in $\mathcal{D}$. For any simply connected domain $\mathcal{D} \subset \mathcal{C}$, we can write $f=h+\bar{g}$, where $h$ and $g$ are analytic in $\mathcal{D}$. We call $h$ the analytic part and $g$ the co-analytic part of $f$. A necessary and sufficient condition for $f$ to be locally univalent and sense preserving in $\mathcal{D}$ is that $\left|h^{\prime}(z)\right|>\left|g^{\prime}(z)\right|$ in $\mathcal{D}$, for more details the reader is referred to $[1,2,6,7,11-15,17,18,29,30]$.

Denoted by $M_{H}(p)$, the class of $p$-valent meromorphic harmonic functions $f$ that are sense preserving in the punctured open unit disc $\mathcal{U}^{*}=\{z \in \mathcal{C}: 0<|z|<1\}=\mathcal{U} \backslash\{0\}$, where $\mathcal{U}=\{z \in \mathcal{C}:|z|<1\}$ and $f$ is of the form

$$
f=h+\bar{g},
$$

where

$$
f(z)=h(z)+\overline{g(z)}=z^{-p}+\sum_{n=1}^{\infty} a_{n+p-1} z^{n+p-1}+\overline{\sum_{n=1}^{\infty} b_{n+p-1} z^{n+p-1}} .
$$

Also, we denote by $M_{\bar{H}}(p)$ the class of $p$-valent (multivalent) meromorphic harmonic function $f \in$ $M_{\bar{H}}(p)$ and is represented by

$$
f(z)=h(z)+\overline{g(z)}=z^{-p}+\sum_{n=1}^{\infty}\left|a_{n+p-1}\right| z^{n+p-1}+\sum_{n=1}^{\infty}\left|b_{n+p-1}\right| \overline{z^{n+p-1}}, \quad\left|b_{p}\right|<1 .
$$

Let $\mathrm{F}$ be a p-valent meromorphic harmonic function given by

$$
\mathrm{F}(z)=\mathrm{H}(z)+\overline{\mathrm{G}(z)}=z^{-\mathrm{p}}+\sum_{n=1}^{\infty}\left|A_{n+p-1}\right| z^{n+p-1}+\overline{\sum_{n=1}^{\infty}\left|B_{n+p-1}\right| z^{n+p-1}}, \quad\left|b_{p}\right|<1 .
$$

The Hadamard product (or convolution) of $F$ and $f$ is defined by

$$
(f * F)(z)=z^{-p}+\sum_{n=1}^{\infty} a_{n+p-1} A_{n+p-1} z^{n+p-1}+\overline{\sum_{n=1}^{\infty} b_{n+p-1} B_{n+p-1} z^{n+p-1}}, \quad\left|b_{p}\right|<1 .
$$

Let $f_{1}$ and $f_{2}$ be two analytic functions in the open unit disk $U=\{z \in \mathcal{C}:|z|<1\}$. We say that function $f_{1}$ is subordinate to $f_{2}$ in $\mathcal{U}$ and write $f_{1}(z) \prec f_{2}(z)(z \in \mathcal{U})$, if there exists a Schwarz function $\omega(z)$, which is analytic in $\mathcal{U}$ with $\omega(0)=0$ and $|\omega(z)|<1(z \in \mathcal{U})$, such that $f_{1}(z)=f_{2}(\omega(z)) z \in \mathcal{U}$. For more details see [9].

In 1959, Sakaguchi [26] introduced and studied the class of starlike function with respect to symmetric point in $\mathcal{U}$. Further investigations into the class of starlike functions with respect to symmetric points can be found in the articles [8, 24, 25, 28, 31-33].

Recently, Ghaffar et. al. [20], introduced and investigated a class of meromorphic starlike functions, $f \in M_{H}(1)$, with respect to symmetric point in circular domain which satisfies the condition

$$
-\frac{2 z f^{\prime}(z)}{f(z)-f(-z)} \prec \frac{1+A z}{1+B z}, \quad \text { where } z \in \mathcal{U}^{*},
$$

and $-1 \leqslant B<A \leqslant 1$.

In 2020, the authors in [19] further generalized the above class using a differential operator $\mathcal{D}_{H}$ in defining a class of meromorphic harmonic univalent functions, $f \in M_{H}(1)$, in Janowski domain that are starlike with respect to symmetric point as,

$$
-\frac{2 \mathcal{D}_{\mathrm{H}} \mathrm{f}(z)}{\mathrm{f}(z)-\mathrm{f}(-z)} \prec \frac{1+\mathrm{Az}}{1+\mathrm{Bz}}
$$


where $-1 \leqslant B<A \leqslant 1$ and $\mathcal{D}_{H} f(z)=z h^{\prime}(z)-\overline{z g^{\prime}(z)}$, or equivalently

$$
\left|\frac{\mathcal{D}_{\mathrm{H}} \mathrm{f}(z)+\frac{\mathrm{f}(z)-\mathrm{f}(-z)}{2}}{\mathrm{BD}_{\mathrm{H}} \mathrm{f}(z)+\mathrm{A} \frac{\mathrm{f}(z)-\mathrm{f}(-z)}{2}}\right|<1, \quad\left(z \in \mathcal{U}^{*}\right) .
$$

For more work on harmonic meromorphic multivalent functions see [3, 5, 16, 21-23, 27, 34] .

Now we shall consider the generalization of this class and define the class $\mathcal{M}_{\mathrm{H}, \mathrm{p}}^{* *}[\mathrm{~A}, \mathrm{~B}]$ as follows. Let $-1 \leqslant B<A \leqslant 1$. Then the function $f \in M_{H}(p)$ is in the class $\mathcal{M}_{H, p}^{* *}[A, B]$ if it satisfies the condition

$$
-\frac{2 \mathcal{D}_{\mathrm{H}} f(z)}{f(z)-f(-z)} \prec \frac{p(1+A z)}{1+B z}
$$

where $\mathcal{D}_{\mathrm{H}} \mathrm{f}(z)=z \mathrm{~h}^{\prime}(z)-\overline{z g^{\prime}(z)}$. Further, we denote $\mathcal{M}_{\overline{\mathrm{H}, \mathrm{p}}}^{* *}[\mathrm{~A}, \mathrm{~B}]$, the subclass of $\mathcal{M}_{\mathrm{H}, \mathrm{p}}^{* *}[\mathrm{~A}, \mathrm{~B}]$, consisting of harmonic meromorphic functions of the form (1.2).

\section{Main results}

In this section we obtain our main results for the functions belonging to these classes like sensepreserving, sufficiency criteria, growth results, convex combination and weighted mean.

Theorem 2.1. Let $\mathrm{f}=\mathrm{h}+\overline{\mathrm{g}}$ and of the form (1.1), if satisfies the condition

$$
\sum_{n=1}^{\infty} \alpha_{n}\left|a_{n+p-1}\right|+\beta_{n}\left|b_{n+p-1}\right| \leqslant 1,
$$

with

$$
\alpha_{n}=\frac{\left|(1-B)(n+p-1)+p \frac{(1+A)\left(1-(-1)^{n+p-1}\right)}{2}\right|}{p(A-B)}
$$

and

$$
\beta_{n}=\frac{\left|(1+B)(n+p-1)-p \frac{(1+A)\left(1-(-1)^{n+p-1}\right)}{2}\right|}{p(A-B)},
$$

then $\mathrm{f}$ is harmonic $\mathrm{p}$-valent and sense-preserving in $\mathrm{U}^{*}$ and $\mathrm{f} \in \mathcal{M}_{\mathrm{H}, \mathrm{p}}^{* *}[\mathrm{~A}, \mathrm{~B}]$.

Proof. To show that $f(z)$ is sense-preserving harmonic mapping in $\mathcal{U}^{*}$, consider

$$
\begin{aligned}
\left|h^{\prime}(z)\right| & >\frac{p}{|z|^{p+1}}-\sum_{n=1}^{\infty}(n+p-1)\left|a_{n+p-1}\right||z|^{n+p-2} \\
& =p-\sum_{n=1}^{\infty} \alpha_{n}\left|a_{n+p-1}\right|=\sum_{n=1}^{\infty} \beta_{n}\left|b_{n+p-1}\right|=\sum_{n=1}^{\infty}(n+p-1)\left|b_{n+p-1}\right||z|^{n+p-2}=\left|h^{\prime}(z)\right|
\end{aligned}
$$

this shows that $f$ is sense-preserving. Now to show that $f \in \mathcal{M}_{H, p}^{* *}[A, B]$, we have to prove that

$$
\left|\mathcal{D}_{\mathrm{H}} f(z)+p \frac{f(z)-f(-z)}{2}\right|-\left|B \mathcal{D}_{\mathrm{H}} f(z)+A p \frac{f(z)-f(-z)}{2}\right| \leqslant 0
$$

For this consider

$$
\left|\mathcal{D}_{H} f(z)+p \frac{f(z)-f(-z)}{2}\right|-\left|B \mathcal{D}_{H} f(z)+A p \frac{f(z)-f(-z)}{2}\right|
$$




$$
\begin{aligned}
& =\mid \sum_{n=1}^{\infty}\left[\left((n+p-1)+p \frac{\left(1-(-1)^{n+p-1}\right)}{2}\right) a_{n+p-1} z^{n+p-1}+((n+p-1)\right. \\
& \left.\left.+p \frac{\left(1-(-1)^{n+p-1}\right)}{2}\right) \overline{b_{n+p-1} z^{n+p-1}}\right]|-| \frac{p(A-B)}{z^{p}} \\
& +\sum_{n=1}^{\infty}\left[\left(B(n+p-1)+A p \frac{\left(1-(-1)^{n+p-1}\right)}{2}\right) a_{n+p-1} z^{n+p-1}\right. \\
& \left.+\left(\mathrm{B}(\mathrm{n}+\mathrm{p}-1)+\mathrm{Ap} \frac{\left(1-(-1)^{\mathrm{n}+\mathrm{p}-1}\right)}{2}\right) \overline{\mathrm{b}_{n+p-1} z^{n+p-1}}\right] \\
& \leqslant \sum_{n=1}^{\infty}\left[\left((n+p-1)+p \frac{\left(1-(-1)^{n+p-1}\right)}{2}\right)\left|a_{n+p-1}\right||z|^{n+p-1}+((n+p-1)\right. \\
& \left.\left.+p \frac{\left(1-(-1)^{n+p-1}\right)}{2}\right)\left|b_{n+p-1}\right||z|^{n+p-1}\right]-\frac{p(A-B)}{z^{p}} \\
& +\sum_{n=1}^{\infty}\left[\left(B(n+p-1)+A p \frac{\left(1-(-1)^{n+p-1}\right)}{2}\right)\left|a_{n+p-1}\right|\left|z^{n+p-1}\right|\right. \\
& \left.+\left(B(n+p-1)+A p \frac{\left(1-(-1)^{n+p-1}\right)}{2}\right)\left|b_{n+p-1}\right|\left|z^{n+p-1}\right|\right] \\
& \leqslant \sum_{n=1}^{\infty}\left[\left|(1+B)(n+p-1)+p(1+A) \frac{\left(1-(-1)^{n+p-1}\right)}{2}\right|\left|a_{n+p-1}\right||z|^{n+p-1}\right. \\
& \left.+\left|(1+B)(n+p-1)+p(1+A) \frac{\left(1-(-1)^{n+p-1}\right)}{2}\right|\left|b_{n+p-1}\right||z|^{n+p-1}\right]-\frac{p(A-B)}{|z|^{p}} \\
& =\frac{p(A-B)}{|z|^{p}} \sum_{n=1}^{\infty}\left[\frac{\left|(1+B)(n+p-1)+p(1+A) \frac{\left(1-(-1)^{n+p-1}\right)}{2}\right|}{p(A-B)}\left|a_{n+p-1} \| z\right|^{n+p}\right. \\
& \left.+\frac{\left|(1+B)(n+p-1)+p(1+A) \frac{\left(1-(-1)^{n+p-1}\right)}{2}\right|}{p(A-B)}\left|b_{n+p-1}\right||z|^{n+p}\right]-\frac{p(A-B)}{|z|^{p}} \\
& \leqslant \frac{p(A-B)}{|z|^{p}}\left\{\sum_{n=1}^{\infty}\left[\alpha_{n}\left|a_{n+p-1}\right|+\beta_{n}\left|b_{n+p-1}\right|\right]-1\right\} \leqslant 0 .
\end{aligned}
$$

Where we have used (2.1) and hence completes the proof.

\subsection{Example}

Cosider the meromorphic $p$-valent function

$$
f(z)=\frac{1}{z^{p}}+\sum_{n=1}^{\infty} \frac{x_{n+p-1}}{\alpha_{n}} z^{n+p-1}+\sum_{n=1}^{\infty} \frac{y_{n+p-1}}{\beta_{n}} \bar{z}^{n+p-1},
$$

such that $\sum_{n=1}^{\infty}\left(\left|x_{n+p-1}\right|+\left|y_{n+p-1}\right|\right)=1$, we have

$$
\sum_{n=1}^{\infty}\left(\alpha_{n}\left|a_{n+p-1}\right|+\beta_{n}\left|b_{n+p-1}\right|\right)=\sum_{n=1}^{\infty}\left(\left|x_{n+p-1}\right|+\left|y_{n+p-1}\right|\right)=1
$$


Thus $f \in \mathcal{M}_{\mathrm{H}, \mathrm{p}}^{* *}[\mathrm{~A}, \mathrm{~B}]$ and above coefficient bounds given in (2.1) are sharp for this function.

Theorem 2.2. Let $\mathrm{f}=\mathrm{h}+\overline{\mathrm{g}} \in \mathrm{M}_{\overline{\mathrm{H}}}(\mathrm{p})$ and is of the form (1.2), then $\mathrm{f} \in \mathcal{M}_{\overline{\mathrm{H}}, \mathrm{p}}^{* *}[\mathrm{~A}, \mathrm{~B}]$ if it satisfies the condition

$$
\sum_{n=1}^{\infty} \alpha_{n}\left|a_{n+p-1}\right|+\beta_{n}\left|b_{n+p-1}\right| \leqslant 1
$$

with $\alpha_{n}$ and $\beta_{n}$ are defined in (2.2) and (2.3).

Proof. The proof is similar to Theorem 2.1, so omitted.

Theorem 2.3. Let $\mathrm{f}=\mathrm{h}+\overline{\mathrm{g}} \in \mathcal{M}_{\overline{\mathrm{H}}, \mathrm{p}}^{* *}[\mathrm{~A}, \mathrm{~B}]$ and is of the form (1.2), $0<|z|=\mathrm{r}<1$. Then

$$
\frac{1}{r^{p}}-\frac{1-B}{A-B} r^{p} \leqslant|f(z)| \leqslant \frac{1}{r^{p}}+\frac{1-B}{A-B} r^{p} .
$$

Proof. Consider

$$
\begin{aligned}
|f(z)| & =\left|\frac{1}{z^{p}}+\sum_{n=1}^{\infty}\left(a_{n+p-1} z^{n+p-1}+\overline{b_{n+p-1} z^{n+p-1}}\right)\right| \\
& \leqslant \frac{1}{r^{p}}+\sum_{n=1}^{\infty}\left(\left|a_{n+p-1}\right|+\left|b_{n+p-1}\right|\right) r^{n+p-1} \leqslant \frac{1}{r^{p}}+r^{p} \sum_{n=1}^{\infty}\left(\left|a_{n+p-1}\right|+\left|b_{n+p-1}\right|\right) .
\end{aligned}
$$

Now from (2.1)

$$
\alpha_{1} \sum_{n=1}^{\infty}\left|a_{n+p-1}\right|+\beta_{1} \sum_{n=1}^{\infty}\left|b_{n+p-1}\right| \leqslant \sum_{n=1}^{\infty} \alpha_{n}\left|a_{n+p-1}\right|+\beta_{n}\left|b_{n+p-1}\right| \leqslant 1,
$$

with some calculations we obtain

$$
\sum_{n=1}^{\infty}\left(\left|a_{n+p-1}\right|+\left|b_{n+p-1}\right|\right) \leqslant \frac{1-B}{A-B}
$$

and so

$$
|f(z)| \leqslant \frac{1}{r^{p}}+\frac{1-B}{A-B} r^{p} .
$$

Similarly, proceeding as above we get,

$$
\begin{aligned}
|f(z)| & =\left|\frac{1}{z^{p}}+\sum_{n=1}^{\infty}\left(a_{n+p-1} z^{n+p-1}+\overline{b_{n+p-1} z^{n+p-1}}\right)\right| \\
& \geqslant \frac{1}{r^{p}}-r^{p} \sum_{n=1}^{\infty}\left(\left|a_{n+p-1}\right|+\left|b_{n+p-1}\right|\right) \geqslant \frac{1}{r^{p}}-\frac{1-B}{A-B} r^{p} .
\end{aligned}
$$

This completes the proof.

Theorem 2.4. Let $\mathrm{f}=\mathrm{h}+\overline{\mathrm{g}}$, and is of the form (1.2), then $\mathrm{f} \in \mathcal{M}_{\overline{\mathrm{H}}, \mathrm{p}}^{* *}[\mathrm{~A}, \mathrm{~B}]$ if and only if

$$
f(z)=\sum_{n=0}^{\infty}\left(\gamma_{n} h_{n+p-1}+\delta_{n} g_{n+p-1}\right)
$$


where $\mathrm{h}_{\mathrm{p}-1}(\mathrm{z})=\frac{1}{z^{\mathrm{p}}}$ and for $\mathrm{n}>1$ we have

$$
h_{n+p-1}(z)=\frac{1}{z^{p}}+\frac{p(A-B)}{\left|(1-B)(n+p-1)+p \frac{(1+A)\left(1-(-1)^{n+p-1}\right)}{2}\right|} z^{n+p-1},
$$

also $\mathrm{g}_{\mathfrak{p}-1}(\mathrm{z})=\frac{1}{z^{\mathrm{p}}}$ and for $\mathrm{n}>1$

$$
g_{n+p-1}(z)=\frac{1}{z^{\mathfrak{p}}}+\frac{p(A-B)}{\left|(1-B)(n+p-1)+p \frac{(1+A)\left(1-(-1)^{n+p-1}\right)}{2}\right|} \overline{z^{n+p-1}},
$$

with $\mathrm{n}=0,1,2,3, \ldots, 1 \geqslant \gamma_{\mathrm{n}} \geqslant 0, \mathrm{p} \geqslant 1,1 \geqslant \delta_{\mathrm{n}} \geqslant 0$ and $\sum_{n=0}^{\infty}\left(\gamma_{n}+\delta_{n}\right)=1$.

Proof. Let us consider

$$
\begin{aligned}
f(z)= & \gamma_{0} h_{p-1}+\delta_{0} g_{p-1}+\sum_{n=1}^{\infty}\left(\gamma_{n} h_{n+p-1}+\delta_{n} g_{n+p-1}\right) \\
= & \left(\gamma_{0}+\delta_{0}\right) \frac{1}{z^{p}}+\sum_{n=1}^{\infty} \gamma_{n}\left(\frac{1}{z^{p}}+\frac{p(A-B)}{\left|(1-B)(n+p-1)+p \frac{(1+A)\left(1-(-1)^{n+p-1}\right)}{2}\right|} z^{n+p-1}\right) \\
& +\sum_{n=1}^{\infty} \delta_{n}\left(\frac{1}{z^{p}}-\frac{p(A-B)}{\mid(1-B)(n+p-1)+p \frac{(1+A)\left(1-(-1)^{n+p-1}\right)}{2} z^{n+p-1}}\right) \\
= & \sum_{n=1}^{\infty}\left(\gamma_{n}+\delta_{n}\right) \frac{1}{z^{p}}+\sum_{n=1}^{\infty} \gamma_{n} \frac{p(A-B)}{\mid(1-B)(n+p-1)+p \frac{(1+A)\left(1-(-1)^{n+p-1}\right)}{2}} z^{n+p-1} \\
& -\sum_{n=1}^{\infty} \delta_{n} \frac{p(A-B)}{\left|(1-B)(n+p-1)+p \frac{(1+A)\left(1-(-1)^{n+p-1}\right)}{2}\right|} \frac{z^{n+p-1}}{\mid}
\end{aligned}
$$

Thus,

$$
\begin{aligned}
& \sum_{n=1}^{\infty}\left\{\alpha_{n}\left(\frac{p(A-B)}{\left|(1-B)(n+p-1)+p \frac{(1+A)\left(1-(-1)^{n+p-1}\right)}{2}\right|} \gamma_{n}\right)\right. \\
& \left.\quad+\beta_{n}\left(\frac{p(A-B)}{\left|(1-B)(n+p-1)+p \frac{(1+A)\left(1-(-1)^{n+p-1}\right)}{2}\right|} \delta_{n}\right)\right\}=\sum_{n=1}^{\infty}\left(\gamma_{n}+\delta_{n}\right)=1-\gamma_{0}-\delta_{0} \leqslant 1,
\end{aligned}
$$

hence $f \in \mathcal{M}_{\overline{\mathrm{H}}, \mathrm{p}}^{* *}[A, B]$, conversely, let $f \in \mathcal{M}_{\overrightarrow{\mathrm{H}}, \mathrm{p}}^{* *}[A, B]$, set

$$
\begin{aligned}
& \gamma_{n}=\frac{\left|(1+B)(n+p-1)+p(1+A) \frac{\left(1-(-1)^{n+p-1}\right)}{2}\right|}{p(A-B)}\left|a_{n+p-1}\right|, \quad 0 \leqslant \gamma_{n} \leqslant 1, p \geqslant 1, \\
& \delta_{n}=\frac{\left|(1+B)(n+p-1)+p(1+A) \frac{\left(1-(-1)^{n+p-1}\right)}{2}\right|}{p(A-B)}\left|b_{n+p-1}\right|, \quad 0 \leqslant \delta_{n} \leqslant 1, p \geqslant 1, \\
& \gamma_{0}=1-\sum_{n=1}^{\infty} \gamma_{n}-\sum_{n=1}^{\infty} \delta_{n} .
\end{aligned}
$$


Therefore $f$ can be written as

$$
\begin{aligned}
f(z)= & \frac{1}{z^{p}}+\sum_{n=1}^{\infty}\left|a_{n+p-1}\right| z^{n+p-1}-\sum_{n=1}^{\infty}\left|b_{n+p-1}\right| \overline{z^{n+p-1}} \\
= & \frac{1}{z^{p}}+\frac{p(A-B)}{\left|(1-B)(n+p-1)+p \frac{(1+A)\left(1-(-1)^{n+p-1}\right)}{2 p-1}\right|} \gamma_{n}\left|a_{n+p-1}\right| z^{n+p-1} \\
& -\sum_{n=1}^{\infty} \frac{p(A-B)}{\left|(1-B)(n+p-1)+p \frac{(1+A)\left(1-(-1)^{n+p-1}\right)}{2 p-1}\right|} \delta_{n}\left|b_{n+p-1}\right| z^{n+p-1} \\
= & \left(\gamma_{0}+\delta_{0}\right) \frac{1}{z^{p}}+\sum_{n=1}^{\infty} \gamma_{n}\left(\frac{1}{z^{p}}+\frac{p(A-B)}{\left|(1-B)(n+p-1)+p \frac{(1+A)\left(1-(-1)^{n+p-1}\right)}{2 p-1}\right|} z^{n+p-1}\right) \\
& +\sum_{n=1}^{\infty} \delta_{n}\left(\frac{1}{z^{p}}+\frac{p(A-B)}{\mid(1-B)(n+p-1)+p \frac{(1+A)\left(1-(-1)^{n+p-1}\right)}{2 p-1}} z^{n+p-1}\right) \\
= & \sum_{n=1}^{\infty}\left(\gamma_{n} h_{n+p-1}+\delta_{n} g_{n+p-1}\right),
\end{aligned}
$$

hence proved.

Theorem 2.5. The class $\mathcal{M}_{\overline{\mathrm{H}}, \mathrm{p}}^{* *}[\mathrm{~A}, \mathrm{~B}]$ is closed under convex combination.

Proof. For $k \in \mathbb{N}=\{1,2,3, \ldots\}$, let $f_{k} \in \mathcal{M}_{\overline{\mathrm{H}}, \mathrm{p}}^{* *}[A, B]$ and are of the form

$$
f_{k}(z)=\frac{1}{z^{p}}+\sum_{n=1}^{\infty}\left(\left|a_{k, n+p-1}\right| z^{n+p-1}-\left|b_{k, n+p-1}\right| \overline{z^{n+p-1}}\right)
$$

then from (2.1), we get

$$
\sum_{n=1}^{\infty} \alpha_{n}\left|a_{n+p-1}\right|+\beta_{n}\left|b_{n+p-1}\right| \leqslant 1,
$$

for $\sum_{k=1}^{\infty} \delta_{k}=1,\left(0 \leqslant \delta_{k} \leqslant 1\right)$ the convex combination of $f_{k}$ is

$$
\sum_{k=1}^{\infty} \delta_{k} f_{k}(z)=\frac{1}{z^{p}}+\sum_{n=1}^{\infty}\left(\sum_{k=1}^{\infty} \delta_{k}\left|a_{k, n+p-1}\right|\right) z^{n+p-1}-\sum_{n=1}^{\infty}\left(\sum_{k=1}^{\infty} \delta_{k}\left|b_{k, n+p-1}\right|\right) \overline{z^{n+p-1}}
$$

Now using (2.1), we have

$$
\begin{aligned}
& \sum_{n=1}^{\infty}\left(\alpha_{n+p-1} \sum_{k=1}^{\infty} \delta_{k}\left|a_{k, n+p-1}\right|+\beta_{n+p-1} \sum_{k=1}^{\infty} \delta_{k}\left|b_{k, n+p-1}\right|\right) \\
& =\sum_{k=1}^{\infty} \delta_{k}\left(\sum_{n=1}^{\infty}\left(\alpha_{n+p-1}\left|a_{k, n+p-1}\right|+\beta_{n+p-1}\left|b_{k, n+p-1}\right|\right)\right) \leqslant \sum_{k=1}^{\infty} \delta_{k}=1,
\end{aligned}
$$

thus the desired result is obtained.

Theorem 2.6. Let $\mathrm{f}_{\mathrm{k}} \in \mathcal{M}_{\overline{\mathrm{H}}, \mathrm{p}}^{* *}[\mathrm{~A}, \mathrm{~B}]$, for $\mathrm{k}=\{1,2\}$ be of the form (2.5), then their weighted mean $\mathrm{F}_{\mathrm{i}}$ is also in the class $\mathcal{M}_{\overline{\mathrm{H}}, \mathrm{p}}^{* *}[\mathrm{~A}, \mathrm{~B}]$, where $\mathrm{F}_{\mathrm{i}}$ is define below

$$
F_{i}(z)=\frac{(1-i) f_{1}(z)+(1+i) f_{2}(z)}{2}
$$


Proof. From (2.6), one may easily write

$$
\begin{aligned}
F_{i}(z)=\frac{1}{z}+\frac{(1-i) f_{1}(z)+(1+i) f_{2}(z)}{2}= & \frac{1}{z}+\sum_{k=1}^{\infty}\left[\frac{(1-j)\left|a_{n+p-1,1}\right|+(1+j)\left|a_{n+p-1,2}\right|}{2} z^{n+p-1}\right. \\
& -\frac{\left.(1-j)\left|b_{n+p-2,1}\right|+(1+j)\left|b_{n+p-1,2}\right| \frac{}{z^{n+p-1}}\right] .}{2}
\end{aligned}
$$

To show that $\mathrm{F}_{\mathrm{i}} \in \mathcal{M}_{\overline{\mathrm{H}}, \mathrm{p}}^{* *}[\mathrm{~A}, \mathrm{~B}]$, it is enough to show that

$$
\sum_{k=1}^{\infty}\left[\left|\frac{(1-j)\left|a_{n+p-1,1}\right|+(1+j)\left|a_{n+p-1,2}\right|}{2}\right| \alpha_{n+p-1}+\left|\frac{(1-j)\left|b_{n+p-1,1}\right|+(1+j)\left|b_{n+p-1,2}\right|}{2}\right| \beta_{n+p-1}\right] \leqslant 1 .
$$

Now consider

$$
\begin{aligned}
\sum_{k=1}^{\infty}[ & \left(\frac{(1-j)\left|a_{n+p-1,1}\right|+(1+j)\left|a_{n+p-1,2}\right|}{2}\left|\alpha_{n+p-1}+\right| \frac{(1-j)\left|b_{n+p-1,1}\right|+(1+j)\left|b_{n+p-1,2}\right|}{2} \mid \beta_{n+p-1}\right] \\
= & \sum_{k=1}^{\infty}\left[\frac{(1-j)\left|a_{n+p-1,1}\right| \alpha_{n+p-1}+(1-j)\left|b_{n+p-1,1}\right| \beta_{n+p-1}}{2}\right. \\
& \left.+\frac{(1+j)\left|a_{n+p-1,2}\right| \alpha_{n+p-1}+(1+j)\left|b_{n+p-1,2}\right| \beta_{n+p-1}}{2}\right] \\
= & \frac{(1-j)}{2} \sum_{k=1}^{\infty}\left(\left|a_{n+p-1,1}\right| \alpha_{n+p-1}+\left|b_{n+p-1,1}\right| \beta_{n+p-1}\right) \\
& +\frac{(1+j)}{2} \sum_{k=1}^{\infty}\left(\left|a_{n+p-1,2}\right| \alpha_{n+p-1}+\left|b_{n+p-1,2}\right| \beta_{n+p-1}\right) \\
\leqslant & \frac{(1-j)}{2}+\frac{(1+j)}{2}=1 .
\end{aligned}
$$

Hence, $\mathrm{F}_{i} \in \mathcal{M}_{\overline{\mathrm{H}, \mathrm{p}}}^{* *}[\mathrm{~A}, \mathrm{~B}]$.

\section{Acknowledgment}

This research is funded by Department of Mathematics, Faculty of Ocean Engineering Technology and Informatics, Universiti Malaysia Terengganu, 21030 Kuala Nerus, Terengganu, Malaysia.

\section{References}

[1] O. P. Ahuja, Planar harmonic univalent and related mappings, J. Inequal. Pure Appl. Math., 6 (2005), 18 pages. 1

[2] O. P. Ahuja, J. M. Jahangiri, Errata to: "Multivalent harmonic starlike functions", Ann. Univ. Mariae Curie-Skłodowska Sect. A, 55 (2001), 1-13. 1

[3] O. P. Ahuja, J. M. Jahangiri, Multivalent meromorphic harmonic functions, Adv. Stud. Contemp. Math. (Kyungshang), 7 (2003), 179-187. 1

[4] A. Aleman, A. Constantin, Harmonic maps and ideal fluid flows, Arch. Ration. Mech. Anal., 204 (2012), 479-513. 1

[5] H. A. Al-zkeri, F. M. Al-Oboudi, On a class of harmonic starlike multivalent meromorphic functions, Int. J. Open Probl. Complex Anal., 3 (2011), 68-81. 1

[6] M. Arif, O. Barkub, H. M. Srivastava, S. Abdullah, S. A. Khan, Some Janowski type Harmonic q-starlike functions associated with symmetrical points, Mathematics, 8 (2020), 16 pages. 1

[7] D. Bshouty, W. Hengartner, M. Naghibi-Beidokhti, p-valent harmonic mappings with finite Blaschke dilatations, Ann. Univ. Mariae Curie-Skłodowska Sect. A, 53 (1999), 9-26. 1

[8] R. Chand, P. Singh, On certain schlicht mappings, Indian J. Pure Appl. Math., 10 (1979), 1167-1174. 1 
[9] J. Clunie, T. Sheil-Small, Harmonic univalent functions, Ann. Acad. Sci. Fenn. Ser. A I Math., 9 (1984), 3-25. 1

[10] O. Constantin, M. J. Martín, A harmonic maps approach to fluid flows, Math. Ann., 369 (2017), 1-16. 1

[11] J. Dziok, On Janowski harmonic functions, J. Appl. Anal., 21 (2015), 99-107. 1

[12] S. Elhaddad, H. Aldweby, M. Darus, On a subclass of harmonic univalent functions involving a new operator containing q-MittagLeffler function, Int. J. Math. Comput. Sci., 14 (2019), 833-847.

[13] J. M. Jahangiri, Coefficient bounds and univalence criteria for harmonic functions with negative coefficients, Ann. Univ. Mariae Curie-Skłodowska Sect. A, 52 (1998), 57-66.

[14] J. M. Jahangiri, Harmonic meromorphic starlike functions, Bull. Korean Math. Soc., 37 (2000), 291-301.

[15] J. M. Jahangiri, H. Silverman, Meromorphic Univalent Harmonic Functions with Negative Coefficients, Bull. Korean Math. Soc., 36 (1999), 763-770. 1

[16] A. Juma, Study of harmonic multivalent meromorphic functions by using generalized hypergeometric functions, J. Nonlinear Anal. Optim., 2 (2011), 367-372. 1

[17] M. G. Khan, B. Ahmad, T. Abdeljawad, Applications of a differential operator to a class of harmonic mappings defined by MittagLeffer functions, AIMS Math., 5 (2020), 6782-6799. 1

[18] M. G. Khan, B. Ahmad, N. E. Cho, Properties of harmonic mappings associated with polylogrithm functions, Applied Mathematics E-Notes, (Accepted). 1

[19] M. G. Khan, B. Ahmad, M. Darus, W. K. Mashwani, S. Khan, On Janowski Type Harmonic Meromorphic Functions with respect to Symmetric Point, J. Funct. Spaces, 2021 (2021), 5 pages. 1

[20] M. G. Khan, M. Darus, B. Ahmad, G. Murugusundaramoorty, R. Khan, N. Khan, Meromorphic starlike functions with respect to symmetric points, Int. J. Anal. Appl., 18 (2020), 1037-1047. 1

[21] H.-G. Li, Some properties of certain meromorphic multivalent close-to-convex functions, Miskolc Math. Notes, 21 (2020), $249-259$. 1

[22] S. Li, H. Tang, L. Ma, E. Ao, A new class of harmonic multivalent meromorphic functions, Bull. Math. Anal. Appl., 7 (2015), 20-30.

[23] G. Murugusundaramoorthy, Starlikeness of multivalent meromorphic harmonic function, Bull. Korean Math. Soc., 40 (2003), 553-564. 1

[24] I. R. Nezhmetdinov, A sharp lower bound in the distortion theorem for the Sakaguchi class, J. Math. Anal. Appl., 242 (2000), 129-134. 1

[25] M. Ozawa, An elementary proof of the Bieberbach conjecture for the sixth coefficient, Kodai Math. Sem. Rep., 21 (1969), $129-132$. 1

[26] K. Sakaguchi, On a certain univalent mapping, J. Math. Soc. Japan, 11 (1959), 72-75. 1

[27] F. M. Sakar, H. O. Guney, New subclass of multivalent meromorphic harmonic functions defined by a new generalized al-boudi differential operator, World Appl. Sci. J., 20 (2012), 1696-1703. 1

[28] T. N. Shanmugam, C. Ramachandran, V. Ravichandran, Fekete-Szegö problem for subclasses of starlike functions with respect to symmetric points, Bull. Korean Math. Soc., 43 (2006), 589-598. 1

[29] T. Shiel-Small , Constants for planar harmonic mappings, J. London Math. Soc., 42 (1990), 237-248. 1

[30] H. Silverman, Harmonic univalent functions with negative coeffcients, J. Math. Anal. Appl., 220 (1998), 283-289. 1

[31] J. Thangamani, On starlike functions with respect to symmetric points, Indian J. Pure Appl. Math., 11 (1980), $392-405.1$

[32] Z.-G. Wang, Some subclasses of close-to-convex and quasi-convex functions, Mat. Vesnik, 59 (2007), 65-73.

[33] Z.-G. Wang, C.-Y. Gao, S.-M. Yuan, On certain subclasses of close-to-convex and quasi-convex functions with respect to k-symmetric points, J. Math. Anal. Appl., 322 (2006), 97-106. 1

[34] A. K. Yadav, Harmonic multivalent meromorphic functions defined by an integral operator, J. Appl. Math. Bioinform., 2 (2012), 99-114. 1 